$>$ Objective: The dysregulation of neuroinflammation is one of the attributes of the pathogenesis of Alzheimer's disease (AD). Over-expression of complement proteins colocalizes with neurofibrillary tangles, thereby indicating that a complement system may be involved in neuro-inflammation. Here, we report the influence of complement activation on the neuro-inflammation using a microglial cell line.

Methods: first, we performed a cytotoxic assay using the microglial cells BV-2. Second, after treatment of BV-2 cells with $A \beta_{42}$ and/ or $\mathrm{C} 5 \mathrm{a}$, the anaphylatoxin derived from $\mathrm{C}$, we determined the expression levels of the pro-inflammatory factors TNF- $\alpha, I L-1 \beta$, and IL-6. Finally, we explored whether this neuroinflammatory response was mediated by JAK/ STAT3 signaling.

Results: C5a had an enhanced effect on the neural cell viability of $B V-2$ cells treated with $A \beta_{42}$. In addition, C5a also increased the $A \beta$-induced neuro-inflammatory response, and these effects were blocked by the C $5 a R$ antagonist, PMX205. Finally, we demonstrated that the neuro-inflammatory responses induced by $A \beta$ and $C 5$ a were mediated through JAK/STAT3 signaling. By blocking this pathway with an antagonist, AG490, the expression of TNF- $\alpha, I L-1 \beta$, and IL- 6 was alleviated.

Conclusion: The complement protein C5a could exaggerate the $A \beta$-induced neuroinflammatory response in microglia, and $C 5 a R$ may be a potential therapeutic tool for $A D$ treatment. <

Key words: Alzheimer's disease; $\beta$-amyloid; complement system; neuro-inflammatory response; STAT3.

\section{Complement protein C5a enhances the $\beta$-amyloid-induced neuro-inflammatory response in microglia in Alzheimer's disease}

Xiao-qun $\mathrm{An}^{1 *}$, Wei $\mathrm{Xi}^{1}$, Chen-yun $\mathrm{Gu}^{1}$, Xiao Huang ${ }^{2 *}$

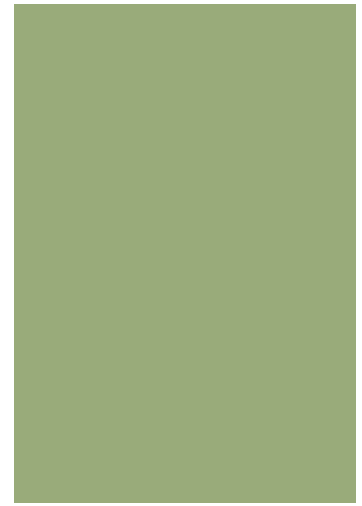

${ }^{1} \mathrm{MD}$, Department of Psychiatry, Yangpu District Mental Health Center of Shanghai, 585 Jungong Road, Shanghai (200090), China. ${ }^{2} \mathrm{MD}$, Department of Psychological Medicine, Zhongshan Hospital, Fudan University, 180 Fenglin Road, Shanghai (200032), China. $\star$ The authors contributed equally to this work. They are co-corresponding authors anxion@163.com

\section{Introduction} huangxia0320@126.com

Alzheimer's disease (AD) is one of the neurodegenerative diseases that frequently occur in elderly populations; it is characterized by cognition impairment and progressive memory loss [1]. An estimated 60 million people suffer from $A D$, and approximately 10 million new cases are diagnosed each year worldwide [2]. Currently, accumulation of fibrillary amyloid $\beta$ (fA $\beta$ ) surrounding the microglial cells and inducing the inflammatory response is believed to be a pathogenesis of AD [3]; microglial cells surrounding the senile plaques are further recruited, and neurotoxic factors (such as IL-1, TNF- $\alpha$, and IL-6) are released. It aggravates the inflammatory responses, although microglial cells normally function as macrophages to help eliminate $A \beta$ [4]. Treatment with $A \beta$ oligomer in primary microglial cells can increase the expressions of IL- $1 \beta$ and TNF- $\alpha$, which are significantly increased in serum and cerebrospinal fluid of $A D$ patients $[5,6]$. Afterward, these inflammatory factors would feed back to the neurons and microglial cells, thereby promoting the production of other inflammatory factors. Hence, a vicious cycle is developed between the microglial cells that are activated by accumulation of $A \beta$ and the inflammatory mediators released by microglial cells, thereby resulting in the denaturation and necrosis of neurons $[7,8]$.

Complement system plays an important role in the identification and removal of invasive pathogenic microorganisms as one part of selfdefense immune system $[9,10]$. Misfolded and aggregated proteins or reactive microglia found in neurodegenerative diseases can activate the complement pathways $[11,12]$. C5a and its receptor (C5aR) 


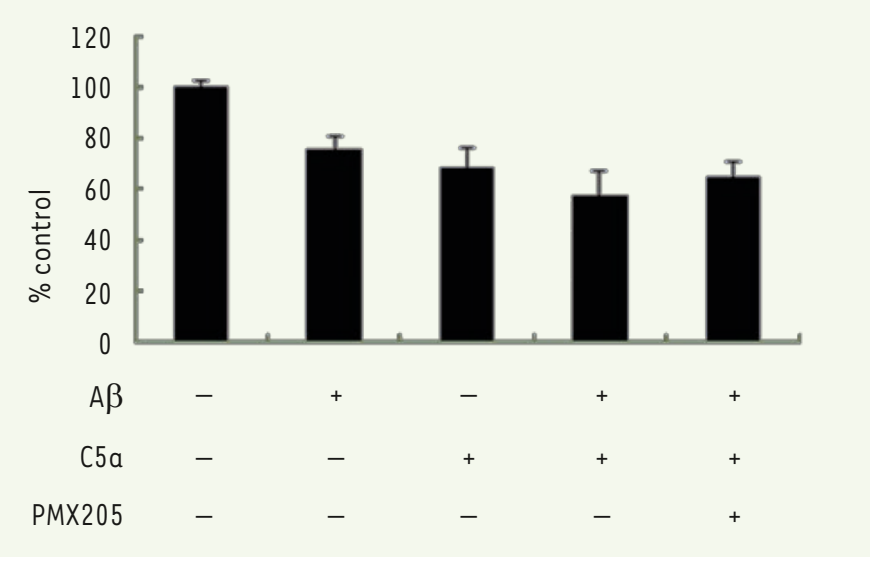

Figure 1. Cell viability was measured by a CCK8 assay after treatment with $A \beta_{42}$, C5a, and PMX205 in BV-2 for $24 h$.

are prominently up-regulated in microglia co-localized with amyloid plaques in AD mouse models [13]. Brain tissues of AD patients revealed an increased expression of two $C 5$ receptors, $C D 88$, and $C 5 \mathrm{~L} 2$, which was associated with abundant neurofibrillary tangles when compared with age-matched counterparts [14]. Continuously activated complement system results in excessive production of $\mathrm{C} 5 \mathrm{a}$ and subsequently exaggerates the neuro-inflammatory response [15]. In addition, C5a enhances the injury of fibrillary amyloid $\beta$ to the primary neurons [16). Hence, blocking the $\mathrm{C} 5 \mathrm{a} / \mathrm{C} 5 \mathrm{aR}$ signaling activation axis alleviates the neuro-inflammatory alterations to $A D$ pathologies. Both $C 5 a-t a r-$ geting vaccines and C5a receptor antagonist, PMX205, have shown improved contextual memory and reduced cerebral amyloid plaque $[17,18]$. However, the underlying mechanisms by which $C 5 a / C 5 a R$ are involved in $A D$ pathogenesis have not been yet elucidated.

Janus kinase-signal transducer and activator of transcription (JAKSTAT) has become one of the important regulatory pathways associated with AD pathogenesis [19]. STAT3, a critical nuclear transcription factor in this pathway, is involved in the neuro-inflammatory response caused by the activation of microglial cells at the onset of $A D$, thereby producing multiple inflammatory factors [20]. C5a was previously reported to induce STAT3 activation [21]. However, the influence of this effect in the context of AD has not been studied. Therefore, we used an $A \beta$ oligomer to induce the in vitro inflammatory response of microglial cells and investigated the role of $\mathrm{C} 5 \mathrm{a} / \mathrm{C} 5 \mathrm{aR}$ pathway in this process [22]. We also tried to explore the effects of C5a-induced STAT3 activation on $A \beta$ oligomer-induced neuro-inflammatory response.

\section{Materials and methods}

\section{Reagents}

Cell culture medium high-glucose Dulbecco's modified eagle medium (DMEM) and fetal bovine serum (FBS) were from Gibco, USA; $0.25 \%$ trypsin and penicillin-streptomycin were from Hyclone, USA; CCK-8 kit was from Dojindo, Japan; $A \beta_{1-42}$ peptides, hexafluoroisopropanol (HFIP) and DMSO were from Sigma, USA; Trizol for extraction of total
RNA and cDNA synthesis kit were from Takara, Japan; ELISA kits of TNF- $\alpha$ and IL- 6 were from eBioscience, USA; AG490 and JNK/STAT3 specific inhibitor were from Sigma, USA; BCA protein assay kit was from Thermo, USA; rabbit anti-p-STAT3 and STAT3 polyclonal antibodies were from Cell Signaling Technology, USA; PVDF membrane and $\varepsilon C L$ kit were from Millipore, Germany.

\section{Cell culturing}

Microglial BV-2 cells were from the Shanghai Cell Bank of Chinese Academy of Sciences. BV-2 cells were cultured in a medium containing DMEM supplemented with $10 \% \mathrm{FBS}$ in the $37^{\circ} \mathrm{C}, 5 \% \mathrm{CO}_{2}$ humid atmosphere incubator.

\section{CCK- 8 proliferation assay}

Microglial cells were inoculated onto a 96-well plate ( $10^{5} /$ well). After $12 \mathrm{~h}, A \beta_{1-42}$ oligomers at different concentrations were added, and BV cells were cultured for another $24 \mathrm{~h}$. The supernatant was replaced with a fresh culture medium, after which $10 \mu \mathrm{L}$ CCK -8 reagent were added to each well and incubated for $2 \mathrm{~h}$. Subsequently, $0 \mathrm{D}_{450}$ values were determined using a microplate reader.

\section{Western blot}

After cell lysis and centrifugation of the cell extracts at $4{ }^{\circ} \mathrm{C}$ for $15 \mathrm{~min}$ at $12,000 \mathrm{rpm}$, the supernatant that contains the cellular proteins was collected and used in Western-blot experiments. $20 \mu \mathrm{g}$ of total proteins were loaded and separated through sodium dodecyl sulfonate polyacrylamide gel electrophoresis (SDS-PAGE) and then transferred onto a PVDF membrane. After incubation in blocking solution for $2 \mathrm{~h}$, the PVDF membrane was probed with primary antibodies to $\mathrm{p}$-STAT3 $(1: 1,000)$, STAT3 $(1: 500)$, and $\beta$-actin $(1: 5,000)$ at $4{ }^{\circ} \mathrm{C}$ overnight. After washing with TBST and probing with the relevant secondary antibodies, an $\varepsilon C L$ kit was used to reveal specific protein expression.

\section{qRT-PCR}

Total RNA was extracted using Trizol according to the manufacturer's instructions. Then, $500 \mathrm{ng}$ total RNA were reverse transcribed into CDNA. PCR conditions: $37^{\circ} \mathrm{C}$ for $15 \mathrm{~min}, 85^{\circ} \mathrm{C}$ for $5 \mathrm{~s}$, and termination at $4^{\circ} \mathrm{C}$ in a $10 \mu \mathrm{L}$ system. The product of cDNA was added into the RT-PCR reaction system as template for the following procedures: initial denaturation for $30 \mathrm{~s}$ at $95^{\circ} \mathrm{C}$; PCR reaction for $5 \mathrm{~s}$ at $95^{\circ} \mathrm{C}$ and $30 \mathrm{~s}$ at $60^{\circ} \mathrm{C}$ followed by 40 cycles; and termination at $95^{\circ} \mathrm{C}$ for $15 \mathrm{~s}, 60^{\circ} \mathrm{C}$ for $1 \mathrm{~min}$, and $95^{\circ} \mathrm{C}$ for $15 \mathrm{~s}$ in $20 \mu \mathrm{L}$ system. The pairs of primer sequences were: STAT-3: (Sense) 5'-TCGTGG 
A

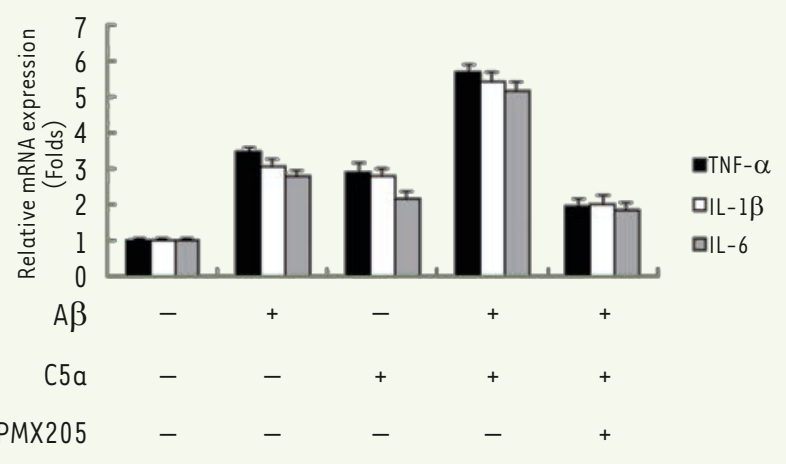

B

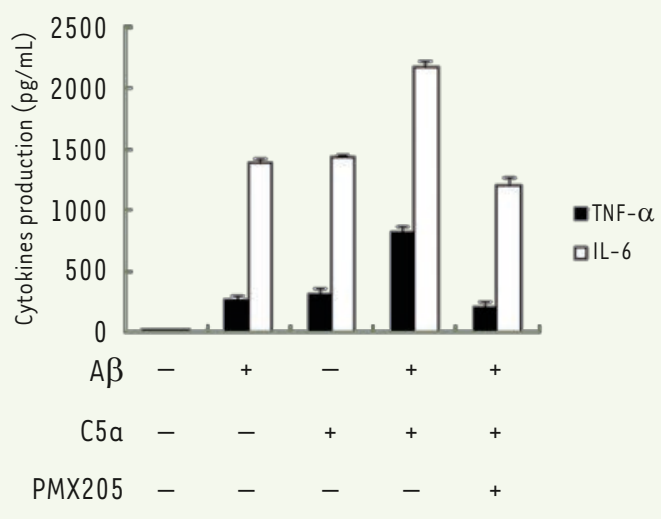

Figure 2. Effects of $C 5 a$ on the inflammatory response stimulated by $A \beta_{42^{*}} A$. mRNA expression levels of TNF- $\alpha, I L-1 \beta$, and IL- 6 were detected by qRT-PCR after cells were treated with $A \beta_{42}, C 5 a$, and PMX205. $B$. Levels of TNF- $\alpha$ and IL-6 in cell culture medium were measured by $\varepsilon$ LISA.

AGCTGTTCAGTTCAGAAAC-3', (Antisense) 5'-GGAAATTTGACCAGCAACCT-3'; IL-1 $\beta$ : (Sense) 5'-GGGCCTCAAAGGAAAGAATC-3', (Antisense) 5'-TACCAGTTGG GGAACTCTGC-3'; TNF- $\alpha$ : (Sense) 5'-TATGGCTCAGGGTC CAACTC-3', (Antisense) 5'-TCCCTTTGCAGAACTCAGG-3'; $\beta$-actin: (Sense) 5'-GTGCTATGTTGCTCTAGA CTTCG-3', (Antisense) 5'-ATGCCACAGGATTCCATACC-3'. All primers were synthesized by Sangon Co., Ltd Biotech (Shanghai, PR China). The transcription level of target gene was evaluated using $2^{-\triangle \Delta c t}$.

\section{Enzyme-linked immunosorbent assay (ELISA)}

After treatment, cell supernatants were collected to quantify the protein expression levels of IL-6 and TNF- $\alpha$ using ELISA detection kits according to the manufacturer's instructions.

\section{Statistical analysis}

Statistical analysis was performed using the SPSS 19.0 software. In the present study, data are presented as mean \pm SEM. Single-factor ANOVA was applied to compare multiple groups. $t$ test was adopted for paired comparison. $P<0.05$ was set as the statistical significance.

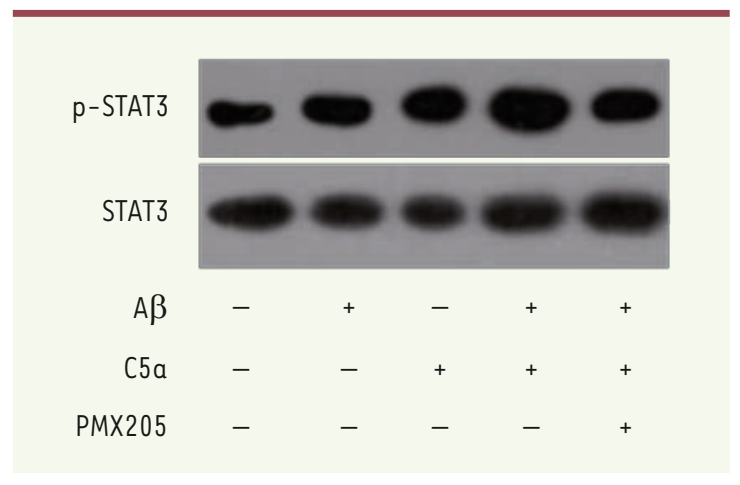

Figure 3. Phosphorylation of STAT3 was detected in both control and treatment groups.

\section{Results}

C5 $\alpha$ enhances the cytotoxic effect of $A \beta_{42}$ on $B V-2$ cells $A \beta_{42}$ and $C 5 a$ inhibited the cell viability of microglia. Thus, we tried to examine the effects of co-treatment with $A \beta_{42}$ and $C 5 a$ on microglia. After a 24 h treatment, the cell viability of BV-2 was tested with a CCK8 assay. As shown in Figure 1, the cell number was reduced by about $50 \%$ in the $A \beta_{42}$ and $C 5$ a co-treatment group compared with $A \beta_{42}$ alone group. Cell growth was restored after adding PMX205.

C5a raises the neuro-inflammatory response to $A \beta_{42}$ To explore the role of $\mathrm{C} 5 \mathrm{a}$ on the inflammatory response in BV-2 cells after exposure to $A \beta_{42}, B V-2$ cells were incubated with $A \beta_{42}, C 5 a, A \beta_{42}$ plus $C 5 a$, or PMX205 for $24 \mathrm{~h}$. The production of inflammatory factors, TNF- $\alpha$, $I L-1 \beta$, and $I L-6$, were analyzed. As shown in Figure $2 A$, the expression of these pro-inflammatory molecules increased in both $A \beta_{42}$ and $C 5 a$ groups and was further up-regulated in $A \beta_{42}$ plus C5a group, whereas their expression was substantially reversed in $A \beta_{42}$ plus PMX205 group. We further measured the secreted TNF- $\alpha$ and IL- 6 with ELISA kits. Similarly, more TNF- $\alpha$ and IL- 6 were produced in the $A \beta_{42}$ plus $C 5$ a group than in the $A \beta_{42}$ or $C 5 a$ alone group and were decreased in the $A \beta_{42}$ plus PMX205 group (Figure 2B).

C5 $a$ enhances the $A \beta_{42}$-induced activation of STAT3 in BV-2 cells

Next, we examined whether STAT3 was involved in the stimulation process of $A \beta_{42}$ and $C 5 a$ in BV-2 cells. Figure 3 shows the increase in the phosphorylation of STAT3 after treatment with either $A \beta_{42}$ or $C 5 a$. This increase was further reinforced in the $A \beta_{42}$ and $C 5 a$ combination group. As expected, blocking C 5 a with PMX205 reduced the activation of STAT3. 
A

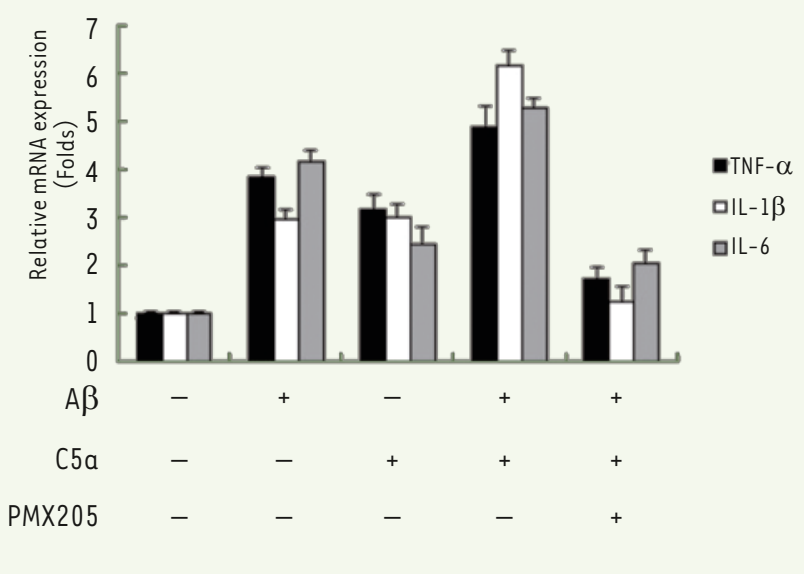

B

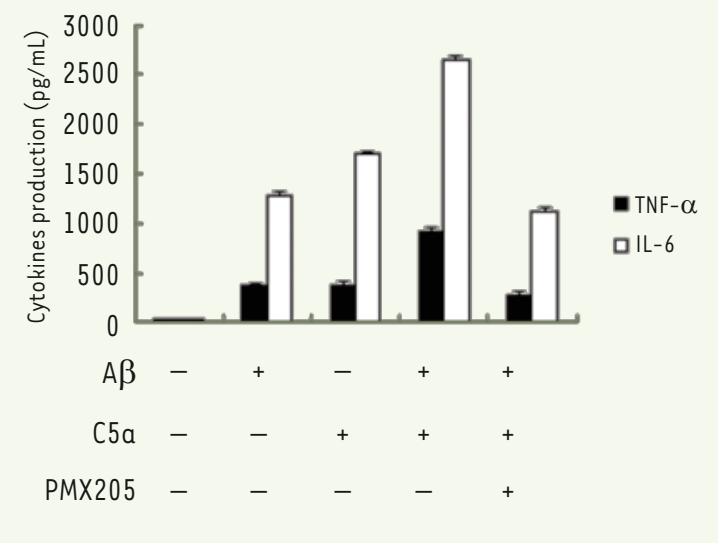

Figure 4. Effects of the pharmacological modulation of STAT3 on inflammatory response stimulated by $A \beta_{42}$ and $C 5 a_{\text {. }}$ A. mRNA expression levels of TNF- $\alpha$, $I L-1 \beta$, and IL- 6 were detected by qRT-PCR after cells were exposed to $A \beta_{42}, C 5 a$, and AG490. B. Levels of TNF- $\alpha$ and IL-6 in cell culture medium were measured by ELISA.

The neuro-inflammatory response to $A \boldsymbol{\beta}_{42}$ and $C 5 a$ is mediated through STAT3 activation

AG490, a STAT3 inhibitor, was used to investigate whether STAT3 activation mediates the neuro-inflammatory response to $A \beta_{42}$ and $C 5 a$. The production of TNF- $\alpha, I L-1 \beta$, and IL- 6 was reduced after treatment with $A G 490$ in cells exposed to $A \beta_{42}$ and $C 5 a$ (Figure $4 A$ ). Moreover, less inflammatory factors were produced when the activation of STAT3 was blocked (Figure 4B).

\section{Discussion}

In the present study, C5a aggravated the cytotoxic effect induced by $A \beta_{42}$ in $B V-2$ cells, consistent with previous findings, in which $C 5 a$ resulted in less $A \beta_{42}$-induced damage to primary neurons isolated from C5a receptor knockout (C5aRIKO) mice [16]. Moreover, C5a enhanced the neuro-inflammatory response stimulated by $A \beta_{42}$ in $B V-2$ cells.
C5aRl, one of two receptors of $C 5 a$, is expressed on the surface of primary microglia isolated from wildtype mice when compared with C5aRl knockout mice. A previous experiment demonstrated that $\mathrm{C}_{5 \mathrm{aRl}}{ }^{+}$cells surrounded $A \beta$ plaques in $A D$ mouse models [13], thereby suggesting that $C 5 a / C 5 a R$ plays a role in the pathogenesis of $A D$. Other studies have reported that the levels of complement components increase in an age-dependent manner, thereby subsequently being able to activate more strongly complement pathways in neurons and microglia [22]. This dysregulation of complement cascade enhances the neuro-inflammatory response to fibrillary $A \beta$ plaques in $A D$ [23]. Hence, approaches to block $\mathrm{C} 5 \mathrm{a} / \mathrm{C} 5 \mathrm{aR}$ activation are hypothesized to help preventing neural damage and cognitive decline. In our study, we confirmed that utilization of C5aRl antagonist, PMX205, restored cell viability to some extent. Moreover, blocking the $\mathrm{C} 5 \mathrm{a} / \mathrm{C} 5 \mathrm{aR}$ interaction had no influence on other complement proteins, such as $\mathrm{Clq}, \mathrm{C} 3$, and $\mathrm{C} 4[13,24]$, thereby suggesting that the benefit of part of the complement system is preserved.

Chronic inflammatory response induced by persistently activated microglia is considered one of the major pathogenesis of AD. C5a, a pro-inflammatory factor, is actively produced after complement activation induced by $A \beta$ deposits, which is one of the major mechanisms for microglia activation $[14,25]$. The activation of microglia and the activation of the complement system further induces neuro-inflammation [23, 26]. Constant production of pro-inflammatory and complement components enhances the release of amyloid peptides [27, 28]. This self-sustaining neuro-inflammation loop between the activated microglia, complement system, and $A \beta$ plaques ultimately results in the loss of synapses and the decline of cognitive function in $A D$ patients $[29,30]$. In the present study, we demonstrated that targeting $\mathrm{C} 5 \mathrm{a}$ and prohibiting its receptor $\mathrm{C} 5 \mathrm{aR}$ obviously suppressed the production of pro-inflammatory factors induced by $A \beta$ oligomers; this finding was consistent with a previous suggestion that $C 5$ aR could be a promising therapeutic target for $A D$.

We further demonstrated that the down-regulation of pro-inflammatory factors via treatment with $\mathrm{C} 5 \mathrm{aR}$ antagonist was mediated through JAK/STAT3 signaling. STAT3, an important nuclear transcription factor of STAT family, mediates the signal transduction of multiple cytokines into the nucleus, thereby subsequently affecting the transcription of target genes and regulating cell function. Increasing evidence supports that the dysregulation of STAT3 is associated with the chronic inflammatory injuries in AD [31]. STAT3 is highly acti- 
vated in brain tissue in AD mouse model [32]. In addition, STAT3 is involved in regulating the transcription of multiple inflammatory factors [19]. Significant decrease in the expression levels of inflammatory factors released by activated microglial cells, such as TNF- $\alpha$ and N0, was observed after the activation of STAT3 was blocked by AG490, a selective inhibitor of JAK signal pathway [33]. Moreover, STAT3 is involved in chronic activation of microglial cells and release of inflammatory factors induced by $A \beta$ oligomers $[34,35]$.

In conclusion, targeting a molecule of the complement system is an effective alternative for $A D$ treatment. Moreover, these results may further improve our understanding of AD pathogenesis, in which chronic neuro-inflammatory response driven by both microglia and complement activation may play a pivotal role. $\diamond$

\section{CONFLICT OF INTERESTS}

Dr. Huang was financially supported by an educational grant from Zhongshan Hospital, Fudan University and Dr. An was financially supported by an grant from Yangpu District Mental Health Center of Shanghai. The authors have no potential conflict of interests.

\section{REFERENCES}

1. Prince M, Bryce R, Albanese $\varepsilon$, Wimo A, Ribeiro W, and Ferri CP. The global prevalence of dementia: a systematic review and metaanalysis. Alzheimer's \& Dementia 2013; 9: 63-75. e2.

2. Prince M, Wino A, Guerchet M, Ali G, Wu Y, and Prina M. World Alzheimer Report 2015. The Global Impact of Dementia: An analysis of prevalence, incidence, cost and trends. 2015. Alzheimer's Disease International, London, 2016.

3. Baranello RJ, Bharani KL, Padmaraju V, Chopra N, Lahiri DK, Greig NH, et al. Amyloid-beta protein clearance and degradation (ABCD) pathways and their role in Alzheimer's disease. Curr Alzheimer Res 2015; 12: 32-46.

4. Latta CH, Brothers HM, and Wilcock DM. Neuroinflammation in Alzheimer's disease; A source of heterogeneity and target for personalized therapy. Neuroscience 2015; 302: 103-11.

5. Wright AL, Zinn R, Hohensinn B, Konen LM, Beynon SB, Tan RP, et al. Neuroinflammation and neuronal loss precede $A \beta$ plaque deposition in the hAPP-J20 mouse model of Alzheimer's disease. PLoS One 2013; 8: e59586.

6. Heneka MT, Kummer MP, and Latz $\varepsilon$. Innate immune activation in neurodegenerative disease. Nat Rev Immunol 2014; 14: 463-77.

7. Heneka MT, Carson MJ, દl Khoury J, Landreth GE, Brosseron F, Feinstein DL, et al. Neuroinflammation in Alzheimer's disease. The Lancet Neurology 2015; 14: 388-405.

8. Serrano-Pozo A, Muzikansky A, Gomez-Isla T, Growdon JH, Betensky RA, Frosch MP, et al. Differential relationships of reactive astrocytes and microglia to fibrillar amyloid deposits in Alzheimer disease. J Neuropathol Exp Neurol 2013; 72: 462-71.

9. Sarma JV and Ward PA. The complement system. Cell and tissue research 2011; 343: 227-35.

10. McGeer PL, Lee M, and McGeer $\varepsilon G$. A review of human diseases caused or exacerbated by aberrant complement activation. Neurobiology of Aging 2016.

11. Crehan H, Hardy J, and Pocock J. Microglia, Alzheimer's disease, and complement. Int J Alzheimers Dis 2012; 2012: 983640.

12. Doens $D$ and Fernandez PL. Microglia receptors and their implications in the response to amyloid beta for Alzheimer's disease pathogenesis. J Neuroinflammation 2014; 11: 48.

13. Ager RR, Fonseca MI, Chu SH, Sanderson SD, Taylor SM, Woodruff TM, et al. Microglial C5aR (CD88) expression correlates with amyloid-beta deposition in murine models of Alzheimer's disease. J Neurochem 2010; 113: 389-401.

14. Fonseca MI, McGuire SO, Counts SE, and Tenner AJ. Complement activation fragment C5a receptors, CD88 and C5L2, are associated with neurofibrillary pathology. J Neuroinflammation 2013; 10: 25.

15. Girke G, Kohl B, Busch C, John T, Godkin 0, Ertel W, et al. Tenocyte activation and regulation of complement factors in response to in vitro cell injury. Mol Immunol 2014; 60: 14-22.

16. Hernandez MX, Namiranian P, Nguyen $\varepsilon$, Fonseca MI, and Tenner AJ. C5a Increases the Injury to Primary Neurons Elicited by Fibrillar Amyloid Beta. ASN Neuro 2017; 9: 1759091416687871.
17. Landlinger C, Oberleitner L, Gruber P, Noiges B, Yatsyk K, Santic R, et al. Active immunization against complement factor $C 5 \mathrm{a}$ : a new therapeutic approach for Alzheimer's disease. J Neuroinflammation 2015; 12: 150.

18. Fonseca MI, Ager RR, Chu SH, Yazan 0, Sanderson SD, LaFerla FM, et al. Treatment with a C5aR antagonist decreases pathology and enhances behavioral performance in murine models of Alzheimer's disease. J Immunol 2009; 183: 1375-83.

19. Nicolas CS, Amici M, Bortolotto ZA, Doherty A, Csaba Z, Fafouri A, et al. The role of JAK-STAT signaling within the CNS. Jak-Stat 2013; 2: e22925.

20. Yin L, Dai Q, Jiang P, Zhu L, Dai H, Yao Z, et al. Manganese exposure facilitates microglial JAK2-STAT3 signaling and consequent secretion of TNF- $\alpha$ and IL-1 $\beta$ to promote neuronal death. NeuroToxicology 2017.

21. Bai B, Horlad H, Saito Y, Ohnishi K, Fujiwara Y, Takeya M, et al. Role of Stat3 activation in cell-cell interaction between B-cell lymphoma and macrophages: the in vitro study. J Clin Exp Hematop 2013; 53: 127-33.

22. Lee WK, Lee SY, Choi JE, Seok Y, Lee $\varepsilon B$, Lee HC, et al. Development of a prognosis - prediction model incorporating genetic polymorphism with pathologic stage in stage I non - small cell lung cancer: A multicenter study. Thorac Cancer 2017; 8: 251-259.

23. Cribbs DH, Berchtold NC, Perreau V, Coleman PD, Rogers J, Tenner AJ, et al. Extensive innate immune gene activation accompanies brain aging, increasing vulnerability to cognitive decline and neurodegeneration: a microarray study. J Neuroinflammation 2012; 9: 179.

24. Wyss-Coray T and Rogers J. Inflammation in Alzheimer disease-a brief review of the basic science and clinical literature. Cold Spring Harb Perspect Med 2012; 2: a006346.

25. Zhou J, Fonseca MI, Pisalyaput $K$, and Tenner AJ. Complement $C 3$ and $C 4$ expression in $\mathrm{Clq}$ sufficient and deficient mouse models of Alzheimer's disease. J Neurochem 2008; 106: 2080-92.

26. Farkas I, Takahashi M, Fukuda A, Yamamoto N, Akatsu H, Baranyi L, et al. Complement $\mathrm{C} 5$ a receptor-mediated signaling may be involved in neurodegeneration in Alzheimer's disease. J Immunol 2003; 170: 5764-71.

27. McGeer $\& G$ and McGeer PL. Neuroinflammation in Alzheimer's disease and mild cognitive impairment: a field in its infancy. J Alzheimers Dis 2010; 19: 355-61.

28. Brandenburg LO, Konrad M, Wruck CJ, Koch T, Lucius R, and Pufe T. Functional and physical interactions between formyl-peptide-receptors and scavenger receptor MARCO and their involvement in amyloid beta 1-42-induced signal transduction in glial cells. J Neurochem 2010; 113: 749-60.

29. Ries M and Sastre M. Mechanisms of Abeta Clearance and Degradation by Glial Cells. Front Aging Neurosci 2016; 8: 160.

30. Stevens B, Allen NJ, Vazquez LE, Howell GR, Christopherson KS, Nouri N, et al. The classical complement cascade mediates CNS synapse elimination. Cell 2007; 131: 1164-78.

31. Schafer DP, Lehrman EK, Kautzman AG, Koyama R, Mardinly AR, Yamasaki $R$, et al. Microglia sculpt postnatal neural circuits in an activity and complement-dependent manner. Neuron 2012; 74: 691-705.

32. Wan J, Fu AK, Ip FC, Ng H-K, Hugon J, Page G, et al. Tyk2/STAT3 signaling mediates $\beta$-amyloid-induced neuronal cell death: implications in Alzheimer's disease. Journal of neuroscience 2010; 30: 6873-81.

33. Ben Haim L, Ceyzeriat K, Carrillo-de Sauvage MA, Aubry F, Auregan G, Guillermier M, et al. The JAK/STAT3 pathway is a common inducer of astrocyte reactivity in Alzheimer's and Huntington's diseases. J Neurosci 2015; 35: 2817-29.

34. Huang C, Ma R, Sun S, Wei G, Fang Y, Liu R, et al. JAK2-STAT3 signaling pathway mediates thrombin-induced proinflammatory actions of microglia in vitro. J Neuroimmunol 2008; 204: 118-25.

35. Xiong J, Wang C, Chen H, Hu Y, Tian L, Pan J, et al. Abeta-induced microglial cell activation is inhibited by baicalin through the JAK2/STAT3 signaling pathway. Int J Neurosci 2014; 124: 609-20.

36. Zhang Y, Li S, He H, Han Q, Wang B and Zhu Y. Influence of Tanshinone IIA on apoptosis of human esophageal carcinoma $\varepsilon c a-109$ cells and its molecular mechanism. Thorac Cancer 2017; 8: 296-303. 